\title{
Vitrifying multiple embryos in different arrangements does not alter the cooling rate
}

\author{
Timothy Ostler ${ }^{\mathrm{a}, *}$, Thomas E. Woolley ${ }^{\mathrm{a}}$, Karl Swann ${ }^{\mathrm{b}}$, Andrew Thompson ${ }^{\mathrm{c}}$, Helen Priddle ${ }^{\mathrm{c}}$, Giles \\ Palmer $^{\mathrm{c}}$, Katerina Kaouri ${ }^{\mathrm{a}}$ \\ ${ }^{a}$ Cardiff School of Mathematics, Cardiff University, Senghennydd Road, Cardiff, CF24 4AG, United Kingdom \\ ${ }^{b}$ Cardiff School of Biosciences, Cardiff University, Museum Avenue, Cardiff, CF10 3AX, United Kingdom \\ ${ }^{c}$ London Women's Clinic Cardiff, 15 Windsor Place, Cardiff, CF10 3BY, United Kingdom
}

\begin{abstract}
Vitrification is the most common method of cryopreservation of gametes in fertility clinics due to its improved survival rates compared to slow freezing techniques. For the Open Cryotop ${ }^{\circledR}$ vitrification device, the number of oocytes, or embryos, mounted onto a single device can vary.

In this work, a mathematical model is developed for the cooling of oocytes, or embryos, that is solved computationally, to investigate whether varying the number of samples mounted onto the Open Cryotop ${ }^{\circledR}$ affects the cooling rates, and therefore survival rates, of vitrified samples. Several realistic spatial arrangements of oocytes/embryos are examined, determining the temperature of the system over time, which highlights the effect of spatial arrangement on the rate of cooling.

Results indicate that neither spatial arrangement nor the number of mounted oocytes, or embryos, has a large effect on cooling rates, so long as the volume of the cryoprotectant remains minimal. Under the manufacturer's guidelines, clinical decisions regarding the number and arrangement of oocytes or embryos placed on a device can be varied, whilst maintaining rapid cooling.
\end{abstract}

Keywords: Mathematical Modelling, Vitrification, Finite Element Methods, Cryopreservation, Heat Transfer, Oocyte, Embryo, Multiple Embryo

\section{Introduction}

Vitrification is 'the supercooling of a liquid to a temperature at which the viscosity is so high that it can be defined as being at a solid state' [1. Vitrification has been used in the application of cryopreservation of gametes and embryos for decades [2, and is now the dominant method of cryopreservation in fertility, taking over from slow freezing methods [3]. High rates of cooling are considered important to the survival of vitrified oocytes and embryos [4, 5, as are high rates of warming during thawing after storage [6]. The process involves the application of permeating cryoprotectants

${ }^{\star}$ This document is the results of the research project funded by the Knowledge Economy Skills Scholarship (KESS2). KESS2 is a pan-Wales higher level skills initiative led by Bangor University on behalf of the HE sector in Wales. It is part funded by the Welsh Government's European Social Fund (ESF) convergence programme for West Wales and the Valleys. The project is additionally funded by, and in collaboration with, the London Women's Clinic. Authors have expressed no conflict of interest or financial affiliation with any of the commercial cryopreservation devices comparedin the study.

${ }^{*}$ Corresponding Author

Email addresses: ostlert@cardiff.ac.uk (Timothy Ostler), woolleyt1@cardiff.ac.uk (Thomas E. Woolley), swannk1@cardiff.ac.uk (Karl Swann), andrew.thomson@londonwomensclinic.com (Andrew Thompson), helen.priddle@londonwomensclinic.com (Helen Priddle), kosmogoniaivf@gmail.com (Giles Palmer), kaourik@cardiff.ac.uk (Katerina Kaouri) 
in high concentrations, which mitigate the risk of formation of ice crystals [1, 7], and direct exposure to liquid nitrogen [8]. A number of different devices and techniques have been developed to facilitate this process, including open pulled straws [9], cryoloops [10, electron microscope grids [1] and the Open Cryotop ${ }^{\circledR}$ device 4 . Vitrified oocytes or embryos are then transferred to storage until needed for an IVF cycle, or until some maximum time period has elapsed. The maximum period depends on national regulations; In the UK, this is governed by the HFEA, who suggest a typical maximum of 10 years [12].

Attempts have been made to study how the construction of various cryopreservation devices affects the rates of temperature change. Methods have been explored to measure cooling rates using thermocouple devices [13, and cooling rates are reported for several devices [9, 14]. An alternative approach to explore heat evolution in such systems is through computational modelling, an established technique with respect to vitrification devices. Such modelling is predominantly aimed at determining system parameters to fit data, or to give theoretical comparisons of different vitrification devices, relating cooling and warming rates to likelihood of embryo and oocyte survival [15, 16, 5.

For some cryopreservation techniques, a single device can have multiple oocytes, or embryos, loaded onto it. For example, the Open Cryotop ${ }^{\circledR}$ (Kitazato ${ }^{\circledR}$ ) may have one to four [17], whilst the SCryolock ${ }^{\circledR}$ device (Biotech Inc.) may carry one or two [18. Recent literature has reported that postthaw survival rates of embryos are affected by the number of embryos vitrified to a single cryo-carrier, but states that there is still further work required to determine some optimum number, and does not give an explanation for this effect [19]. Additionally, when multiple oocytes, or embryos, are loaded onto a single device, it has been observed that they can either remain isolated from one another, or arrange themselves in a more complex geometry. This lack of standardisation of spatial arrangements of samples leads to variability in practice between clinics, operators and even differences between procedures performed by the same operator. Thus, a question arises on whether such differences have any clinical significance, with respect to the survival of the samples. As far as the authors are aware, there is currently no research considering the influence of the number of plated samples and/or their spatial arrangement on the cooling rates of the vitrification process.

The objective of this study is to investigate whether the number of oocytes, or embryos, in addition to their spatial arrangement on the plate, affects cooling rates during vitrification, through the application of computational models. In particular, this study focuses on the Open Cryotop ${ }^{\circledR}$ device, with the vitrification protocol defined according to the user manual [17], although the approach taken in this study can be generalised for other similar devices. Vitrification is considered only of embryos prior to hatching, so the modelled oocytes and embryos are approximately the same size. Hence the term 'sample' is used interchangeably to describe both (see Section 2.2.2).

\section{Materials and Methods}

\subsection{Mathematical Model of vitrification on the Open Cryotop ${ }^{\circledR}$ device}

The Open Cryotop ${ }^{\circledR}$ device consists of a plate with a depth of $0.1 \mathrm{~mm}$ and a width of $0.7 \mathrm{~mm}$, and is made of polypropylene (see Figure 11] [20]. A sample, suspended in a cryoprotectant solution, is pipetted onto the end of the plate (Figure 2), and the entire construction is subsequently submerged into a liquid nitrogen bath.

A schematic diagram of the model is presented in Figure 1. When modelling multiple samples, the samples are placed on the plate at specific locations. Then, cryoprotectant droplets are constructed around each sample, ignoring any overlap between drops (see Figure 3, where a case with two droplets is constructed using COMSOL Multiphysics 5.5). Thus, the cumulative volume of cryoprotectant does not grow in proportion to the number of samples vitrified, as the volume of overlap between droplets depends on how closely the samples are placed to one another and in what pattern. In this way, clinical practice is replicated qw excess cryoprotectant is aspirated, rather than transferring each sample in a fixed volume. 


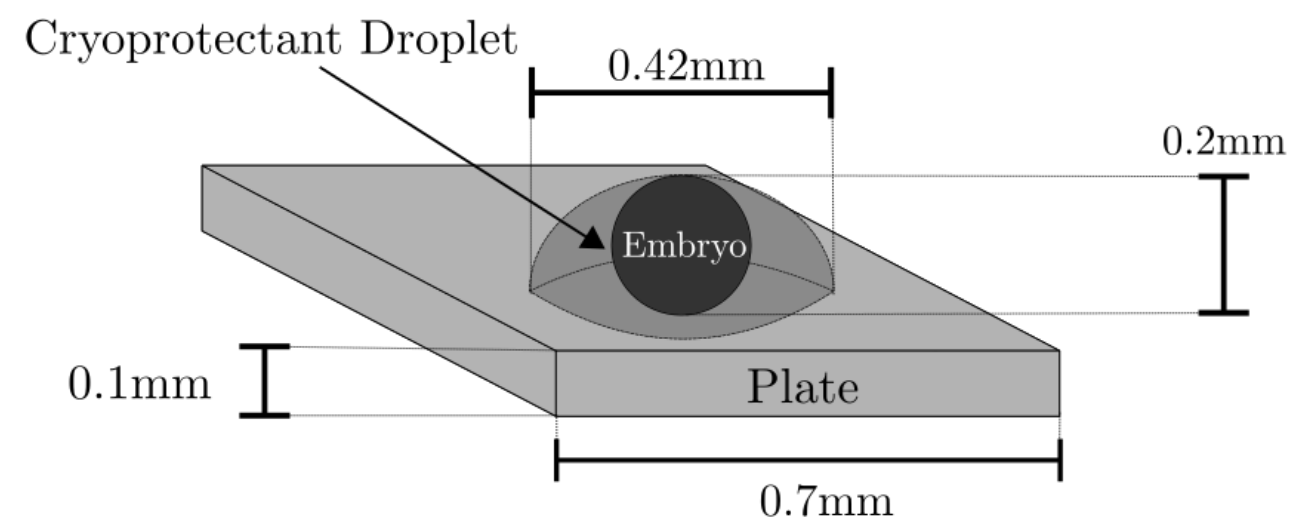

Figure 1: A schematic diagram of the thermal model, including dimensions of the three key domains: the embryo, the plate and the cryoprotectant droplet. The entire system is submerged in liquid nitrogen.

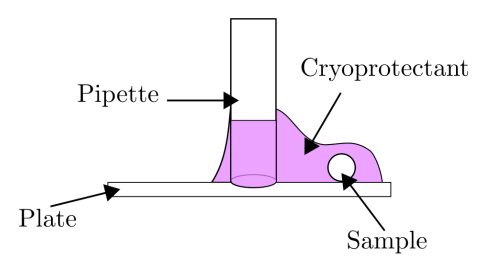

Embryo/oocyte placed onto plate.

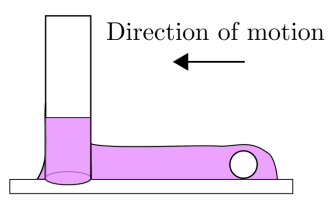

Pipette dragged back across plate.

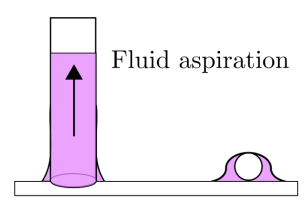

Excess cryoprotectant aspirated, leaving minimal volume droplet.

Figure 2: Diagram depicting the process of transferring a sample from a dish onto the plate of the Open Cryotop ${ }^{\circledR}$, and removing excess cryoprotectant through aspiration to leave a 'minimal droplet'. Adapted from the Open Cryotop ${ }^{\circledR}$ user manual [17.

Once the device, cryoprotectant droplet and sample are constructed, the temperature of each component can be determined over time by solving the heat equation for the system. The heat equation for conductance is used [21], which states that the temperature $u(\boldsymbol{x}, t)$ of a component at some location $\boldsymbol{x}$ and time $t$ satisfies the partial differential equation,

$$
\frac{\partial u(\boldsymbol{x}, t)}{\partial t}=D \nabla^{2} u(\boldsymbol{x}, t)
$$

with $\nabla^{2}$ representing the second spatial derivative, and with an initial temperature of $u(\boldsymbol{x}, 0)=$ $310.15 \mathrm{~K}$. The 'diffusion coefficient', $D$, of the material described by $u$, is a positive constant defined by the relation [21]

$$
D=\frac{k}{\rho c},
$$

for the chemical properties associated to the material of $u$,

- Specific heat capacity $c(\mathrm{~J} /(\mathrm{kg} \mathrm{K}))$,

- Conductivity $k(\mathrm{~W} / \mathrm{mK})$,

- Density $\rho\left(\mathrm{kg} / \mathrm{m}^{3}\right)$.

Additionally, the rate at which liquid nitrogen cools the boundaries of the model where it is in contact 

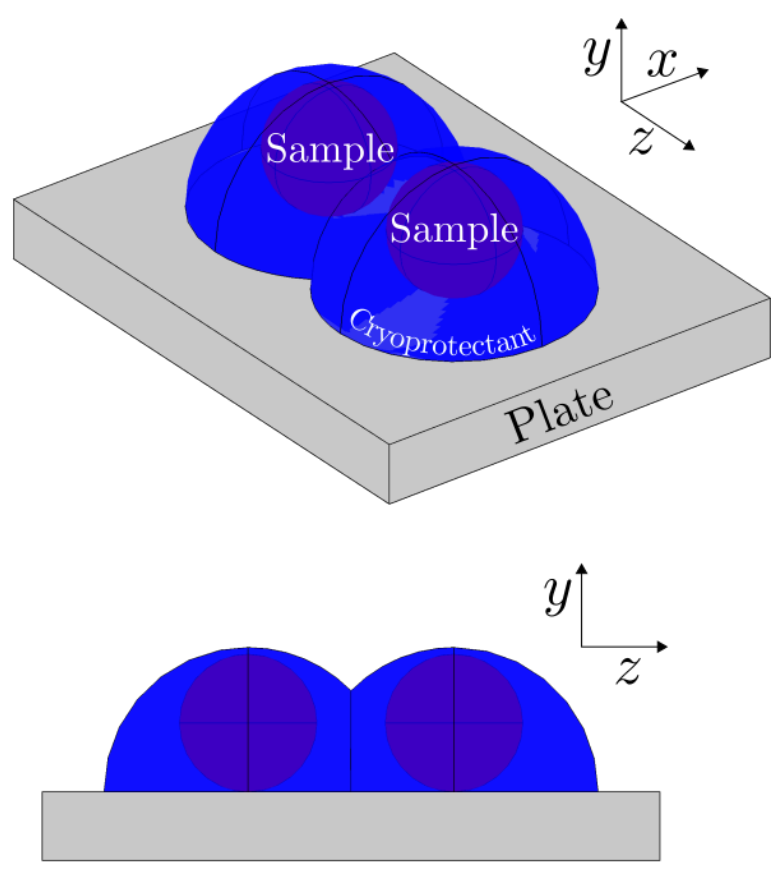

Figure 3: The COMSOL rendering of the fused droplets, with the embryos removed. The same setup is displayed from two different angles.

may be described via a convective heat flux term[21,

$$
-k \frac{\partial u(\boldsymbol{x}, t)}{\partial n}=h\left(u-u_{\mathrm{ext}}\right)
$$

where $u_{\text {ext }}$ is the temperature of the liquid nitrogen.

This approach to model construction and mathematical formulation is similar to those in other literature [15].

The model incorporates the following domains:

- Liquid nitrogen, $L n$,

- The cryoprotectant fluid, F, which consists of ethylene glycol,

- Samples, $S$, which is a disconnected set of spherical samples, also consisting of ethylene glycol,

- The polypropylene plate, $P$.

Where two domains, $A$ and $B$, share a boundary, this boundary is denoted by $\partial \Omega_{A, B}$. A cross section of the model is displayed in Figure 4 with labeled domains and boundaries.

Let $u_{P}(\boldsymbol{x}, t), u_{F}(\boldsymbol{x}, t)$ and $u_{S}(\boldsymbol{x}, t)$ be the temperatures in the plate, cryoprotectant fluid and the samples, respectively, at location $\boldsymbol{x}$ and time $t$. The temperature in the system is described by the 


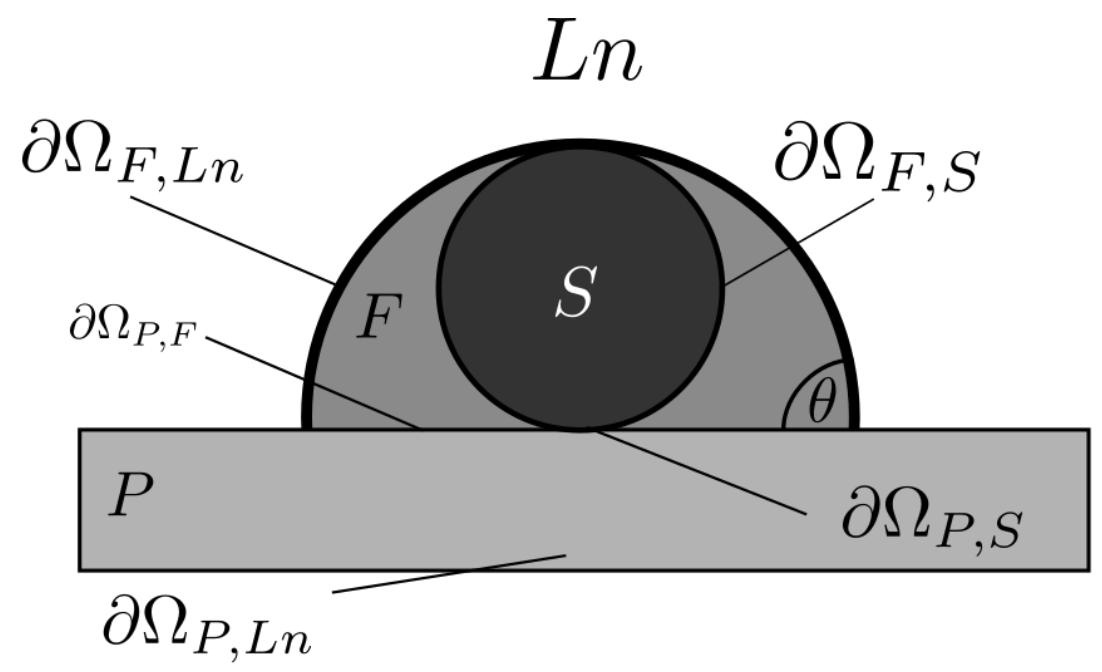

Figure 4: A cross section of the model geometry.

following system of equations:

$$
\begin{aligned}
\frac{\partial u_{P}}{\partial t} & =D_{P} \nabla^{2} u_{P} \quad \text { in } P, \\
\frac{\partial u_{F}}{\partial t} & =D_{F} \nabla^{2} u_{F} \quad \text { in } F, \\
k_{S} \frac{\partial u_{S}}{\partial t} & =D_{S} \nabla^{2} u_{S} \quad \text { in } S, \\
k_{P} \frac{\partial u_{P}}{\partial n} & =h\left(u_{P}-u_{\text {ext }}\right) \quad \text { on } \partial \Omega_{P, L n}, \\
k_{F} \frac{\partial u_{F}}{\partial n} & =h\left(u_{F}-u_{\text {ext }}\right) \quad \text { on } \partial \Omega_{F, L n}, \\
k_{S} \frac{\partial u_{S}}{\partial n} & =k_{F} \frac{\partial u_{F}}{\partial n} \quad \text { on } \partial \Omega_{S, F}, \\
k_{S} \frac{\partial u_{S}}{\partial n} & =k_{P} \frac{\partial u_{P}}{\partial n} \quad \text { on } \partial \Omega_{S, P}, \\
k_{P} \frac{\partial u_{P}}{\partial n} & =k_{F} \frac{\partial u_{F}}{\partial n} \quad \text { on } \partial \Omega_{P, F}, \\
u_{P}(\boldsymbol{x}, 0)=u_{F}(\boldsymbol{x}, 0) & =u_{S}(\boldsymbol{x}, 0)=310.15 \mathrm{~K}, \\
u_{\mathrm{ext}} & =77.15 \mathrm{~K},
\end{aligned}
$$

where Equations (2)-(4) describe heat movement within individual domains, Equations (5) and (6) describe heat convection out of the system through the boundaries of the plate and cryoprotectant into the liquid nitrogen, Equations (7)-(9) describe conduction between the samples and cryoprotectant, samples and plate, and plate and cryoprotectant, respectively, while Equations (10) and (11) describe the initial temperature and external temperature, respectively.

\subsection{Assumptions}

Developing a mathematical model is a highly complex task and requires a number of simplifying assumptions that allow the representation of a physical reality as mathematical objects. Modelling assumptions made in this study are discussed in the following sections to highlight their validity.

\subsubsection{Liquid nitrogen is a stationary, isothermal liquid}

Liquid nitrogen is assumed to be a stationary, isothermal liquid with a fixed temperature of $77.15 \mathrm{~K}$. Hence, the fluid properties of the liquid nitrogen are not modelled in detail. This assumption is 
frequently used in other approaches to the problem [5], and is valid when the device is submerged far beneath the surface of the liquid nitrogen such that it is unaffected by bubble formation and bursting in the liquid nitrogen. Furthermore, the volume of the liquid nitrogen is much greater than the volume of the Open Cryotop ${ }^{\circledR}$ device, thus energy transferred from the device to the liquid nitrogen is quickly spread throughout the system, so near the device the temperature remains constant. Fluid motion would increase the rate of cooling, thus these results offer an upper estimate of the times taken for vitrification. Hence, they offer conservative insights from a "worst case" scenario.

\subsubsection{Samples are spheres of radius $0.1 \mathrm{~mm}$}

Oocytes can vary in size and shape, but are typically spherical, with a diameter of approximately of $0.12 \mathrm{~mm}$ [22. Including the Zona Pellucida and perivitelline space, the total system diameter is approximately $0.15 \mathrm{~mm}$ [23. Cumulus cells are not included, as these are required to be removed according to the Open Cryotop ${ }^{\circledR}$ user manual [17]. Uniform embryos are considered, as deviation from a spherical shape is considered a form of dysmorphia [24]. The choice of $0.1 \mathrm{~mm}$ is a conservative size estimate, and accounts for loss of water volume and artificial collapse during the vitrification process. By choosing a smaller size of oocyte, smaller volumes of cryoprotectant can be used in order to keep the total volume of the droplets minimal.

Since the size of the Zona Pellucida does not change throughout the early stages of the development of an embryo, the same model may be used to describe oocytes and embryos. This makes the assumption that the structural changes occurring post fertilisation do not affect the thermal properties of the embryo, compared to the oocyte.

Assuming a conservative size estimate for samples allows more samples to fit together in dense arrangements. More dense arrangements are more likely to give slower cooling rates, so this assumption also offers conservative insights in the time taken for vitrification.

\subsubsection{Parameter Values}

To construct a numerical model, parameter values must be chosen for $c, k$ and $\rho$ for the plate and cryoprotectant. Defining these values is not a trivial task, as they are 'characteristic of, and measured by, different experimental situations' [21], and can only be accurate if measured for the specific scenario in question. As such, it is necessary that some simplifying assumptions are made, as can be seen in previous literature [15, 13], where the fluid being vitrified is assumed to be water. The vitrification solution contains $15 \%$ ethylene glycol and dimethyl sulphoxide (DMSO) each, as well as a 0.5M sugar solution [20], meaning that although water is present in the solution, the solution is concentrated with cryoprotectants. Hence, it is assumed the thermal properties of the fluid are similar to those of one of the cryoprotectants, particularly ethylene glycol, as the solution is unlikely to behave identically to water.

Another parameter of interest is the surface heat transfer coefficient, $h$, which along with conductivity, affects the rate of heat transfer at the boundary where the contact surface is not between two conductive solids. In particular, this parameter is defined by rate at which the droplet and the plate transfer heat to the surrounding liquid nitrogen. Some research already explores the values $h$ may take in various cryopreservation systems. For example, [16] suggests that plastic straw freezing based methods have a nucleate boiling regime, leading to an estimation of $h=1355 \mathrm{~W} / \mathrm{m}^{2} \mathrm{~K}$, whearas [15] predict that this value should be $1800-2200 \mathrm{~W} / \mathrm{m}^{2} \mathrm{~K}$ for warming of the device in sucrose solution with loading volumes of 0.1 and $0.2 \mu \mathrm{l}$, respectively. These values are all of a similar order of magnitude, but [25] suggests that for freezing of the Cryotop device, $h=9000-10,000 \mathrm{~W} / \mathrm{m}^{2} \mathrm{~K}$ [25], which is on a much larger order of magnitude. Other literature 26] explores how, in a numerical model, different cooling regimes within the liquid nitrogen as a result of fluid motion and phase change might be described by different values of $h$. Choosing values of $h$ that are determined experimentally can validate the assumption 2.2.1, that liquid nitrogen is a stationary domain, as the reduction in cooling rates from this assumption are mitigated. 
A mechanism to help us understand the effects of the choice of $h$ is the 'characteristic timescale', which describes how long it takes heat to diffuse throughout a homogeneous material, whilst $h$ describes the rate at which the boundary of the material changes temperature. By comparing the characteristic timescale to the overall timescale of the cooling process, it can be determined which of the two processes most significantly determines the timescale of the cooling process. When $h$ is large, the characteristic timescale dominates the overall time required for diffusion of heat, but when $h$ is small the rate of boundary warming may be slower than the rate of heat diffusion inside the material, so boundary warming dominates cooling rates.

The characteristic timescale is denoted by

$$
\tilde{t}=\frac{L^{2}}{D}
$$

where $L$ is the 'characteristic length' of a system, $D$ is the associated diffusion coefficient and $\tilde{t}$ is the timescale. Taking the characteristic length to be the droplet radius, $L=0.21 \mathrm{~mm}$, and using the $D$ value for ethylene glycol in Table 1, a timescale of approximately $\tilde{t}=0.14$ seconds is reported. This characteristic time is not dependent on $h$, but the cooling rate is [15]. If $\tilde{t}$ is the dominant timescale in the vitrification process, cooling rates on the order of $100,000 \mathrm{~K}$ per minute will be observed, which is much higher than anything modelled in the Open Cryotop ${ }^{\circledR}$ [15, 5], suggesting that the choice of $h$ is an important consideration in determining cooling rates in this model.

As such, $h=1850 \mathrm{~W} / \mathrm{m}^{2} \mathrm{~K}$ is chosen, which is the lowest value reported in [15] and in line with the value of $h$ from [5] that matches experimental cooling rates from [27]. A low choice of $h$ gives lower cooling rates at the boundary, which gives a conservative estimate of the time taken to vitrify. It may be noted that although this choice of $h$ should be appropriate, the cooling rates in [27] are calculated for a change in temperature from $293.15 \mathrm{~K}$ to $153.15 \mathrm{~K}$. Assuming the temperature can be expressed as separable functions of space and time, in the form $u(\boldsymbol{x}, t)=X(\boldsymbol{x}) T(t)$ (as seen in many solutions in[21]), the function $T(t)$ takes the form of an exponential decay, which means the vitrification timescale does not scale in proportion to the size of the temperature difference. Hence, cooling rates reported by the model may be smaller than those cited from literature, so are conservative estimates.

Referenced literature values chosen for the model are listed in Table 1. Here, the assumption is made that chemical properties are constants, and do not vary with time, space or temperature.

\begin{tabular}{|c|c|c|c|}
\hline Material & Parameter & Value & Source \\
\hline Ethylene Glycol & $\rho$ & $1.11 \times 10^{3} \mathrm{~kg} / \mathrm{m}^{3}$ & COMSOL \\
\hline & $c$ & $3140 \mathrm{~J} / \mathrm{kg} \mathrm{K}$ & COMSOL \\
\hline & $k$ & $2.58 \times 10^{-1} \mathrm{~W} / \mathrm{mK}$ & COMSOL \\
\hline & $D$ & $7.40 \times 10^{-8} \mathrm{~m}^{2} / \mathrm{s} \mathrm{K}$ & COMSOL \\
\hline & $\rho$ & $9.46 \times 10^{2} \mathrm{~kg} / \mathrm{m}^{3}$ & {$[28]$} \\
\hline Polypropylene & $c$ & $1900 \mathrm{~J} / \mathrm{kg} \mathrm{K}$ & {$[28]$} \\
\hline & $k$ & $1.50 \times 10^{-1} \mathrm{~W} / \mathrm{mK}$ & {$[28]$} \\
\hline & $D$ & $8.35 \times 10^{-8} \mathrm{~m}^{2} / \mathrm{s} \mathrm{K}$ & {$[28]$} \\
\hline Liquid nitrogen boundary & $h$ & $1850 \mathrm{~W} / \mathrm{m}^{2} \mathrm{~K}$ & {$[15]$} \\
\hline
\end{tabular}

Table 1: The thermal properties for the materials involved in the system.

\subsubsection{Cryoprotectant droplets are hemispheres with radius $0.21 \mathrm{~mm}$.}

Graphics from the user manual of the Open Cryotop ${ }^{\circledR}$, shown in Figure 2, depict one possible shape of the droplets as being concave following the aspiration of excess media. Additionally, the motion of sliding the pipette back may have an effect on the shape of the droplet. In this work, aspiration and pipette motion are ignored, and assume the droplets to be spherical caps, as spheres minimise the surface tension in a droplet as a result of the isoperimetric inequality (as stated in [29]) in three 
dimensions. Observations suggest spherical caps are a good shape, however, as there is low adhesion of the cryoprotectant to the plate, and small volumes relative to high surface tension appear to force the droplet back into a sphere after the pipette is removed.

The contact angle of the droplet must also be described. The contact angle, $\theta$, is the angle formed between the plate and the tangent plane to the sphere surface at the point of contact, such that $\theta \in(0, \pi)$. At this point there is contact between three domains: the liquid droplet, $L$, the solid plate, $S$, and the liquid nitrogen, $L n$. A tension force $\gamma_{A, B}$ exists between each pair of domains, $A$ and $B$, and these tension forces may be used to define contact angle through a relation known as Young's Equation [30],

$$
\gamma_{S, L n}-\gamma_{S, L}-\gamma_{L, L n} \cos \theta=0
$$

The tension forces depend on the chemical properties of each domain, and environmental factors such as temperature, making it difficult to model accurately. It is observed in the lab that the contact angle is at most $\pi / 2$, as the droplets do not appear truncated at their base. Additionally, the droplet acts structurally as a solid during vitrification [1], so the contact angle does not change as a function of temperature and thus may be fixed as a constant. The droplet must have a height greater than $0.2 \mathrm{~mm}$ above the plate in order to cover the samples, and for a fixed height of droplet, droplet volume decreases with increasing $\theta$. A minimal loading volume is desired such that decrease of the cooling rates from increased droplet volume is minimal, so the model takes $\theta=\pi / 2$. A radius as $0.21 \mathrm{~mm}$ is a valid choice, as it may contain a sample and has a volume of $0.019 \mu \mathrm{l}$, which is within the accepted limit of $0.1 \mu \mathrm{l}$ maximal volume prescribed by the Open Cryotop ${ }^{\circledR}$ manual [17]. Similar modelling approaches in literature use a droplet whose volume is equal to $0.1 \mu \mathrm{l}$ [15], but choosing a smaller volume allows the number of samples used to increase without exceeding this upper volume limit.

\subsubsection{Samples adopt the chemical properties of the cryoprotectant solution}

During vitrification, much of the water is removed from the sample by osmosis, falling as low as $5 \%$ within two minutes [7, and by artificial collapse [31. As such, the sample takes on some volume from the surrounding cryoprotectant solution, so the chemical composition of the sample becomes more similar to that of the surrounding solution [32]. This assumption by highlighting that the sample has a very small volume, so unless samples act as strong insulators, or conductors, any change invoked by this assumption is minimal. Indirectly, this assumption is made by previous literature, which models only a droplet without a suspended sample [15, 5].

\subsection{Spatial arrangements}

When constructing spatial arrangements of samples, samples are placed such that they just touch each other, or there is only a very small separation (see Figure 5), so the effect of tightly packing samples together can be tested. Placing samples as close to each other as possible also maximises the overlap between the separate droplets formed around each sample, which ensures extra cryoprotectant needed to cover both samples is minimal.

The first case studied is a single sample, providing a reference point for more complicated geometries. More samples are then added in a line running down the length of the plate, up to three, to study the effect of increasing the number of samples (see Figure 5a). The case is then studied in which instead of forming a cryoprotectant droplet from the union of the droplets around it, the transfer of multiple samples leads to a small increase in the cumulative volume of droplets merging together, and hence samples are in a 'cylinder' of cryoprotectant with rounded ends (Figure 5b). This scenario tests how minor perturbations in droplet geometry might alter cooling rates.

Additionally, scenarios are studied where the samples are not aligned along the center of the plate, but instead congregate into more complex geometries, which is sometimes observed when multiple samples are vitrified. Three different arrangements are considered, a triangle (Figure 5c), a square (Figure 5d) and a cross (Figure 5e). In practice the actual locations of the samples on the plate may 
vary, but scenarios for every possible location of the samples cannot be constructed. As such, only some of the most densely packed scenarios possible are approached.

In the triangle case, samples are placed such that their centers lie on the corners of an equilateral triangle with side length $0.2 \mathrm{~mm}$. In the computational model, this may cause issues where COMSOL cannot efficiently generate a mesh around the contact points, which appear to overlap. As such, in practice the leftmost sample is shifted (see Figure 5c) along the length of the plate by a small perturbation to ensure enough space exists between it and the other samples, so the formation is now isosceles. In the square case, sample centers form the corners of a square whose side lengths are $0.2 \mathrm{~mm}$. In the cross case, a scenario similar to that of the square is desired, but with a sample in the center. This would cause the droplet to hang off of the plate, however, so instead samples are placed on the corners of a rectangle with side lengths $0.28 \mathrm{~mm}$ wide by $0.3 \mathrm{~mm}$ long. This gives enough space to place a sample in the center of the rectangle. The measurements shown in Figure 5 are calculated based off of these rules.

The cross arrangement is actually not considered a valid arrangement within an IVF clinic due to having 5 samples, but this scenario is tested nonetheless because it allows interesting conclusions to be drawn about what happens to a sample when it is surrounded on all sides by other samples. This means that only the top of the droplet is exposed to liquid nitrogen, and as such represents the greatest risk possible to the sample at the center of the model. This allows commentary on the validity of the manufacturer guidelines suggesting a maximum of four samples per device, with respect to thermal risk.

The final scenario considered is the 'benchmark' scenario, in which the droplet has radius equal to the width of the plate (Figure $5 f$ ), and contains only a single sample at its center. The volume of this droplet is $0.09 \mu \mathrm{l}$, which is just less than the recommended maximal volume of $0.1 \mu \mathrm{l}$, and as such, this represents a case which is known to be safe as it fits within current guidance. Whilst the model cannot necessarily state a minimum safe, or effective, cooling rate, it can use this benchmark to suggest that any scenario whose cooling rate is lower than that of the benchmark cooling rate is deemed unsuitable.

Model code is available on Github.

\footnotetext{
${ }_{1}^{\text {https://github.com/OstlerT/MultipleEmbryoModels }}$
} 


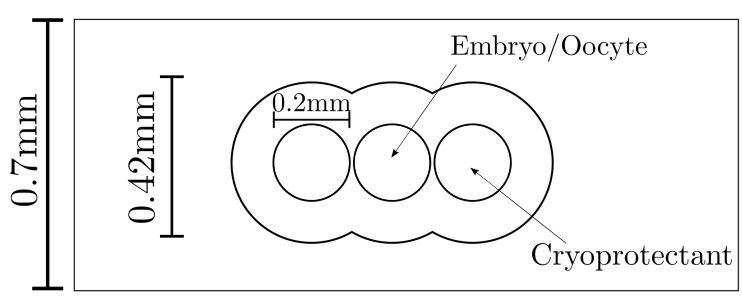

(a) Line. Embryos are spheres inside hemispherical caps of cryoprotectant, added consecutively in a line.

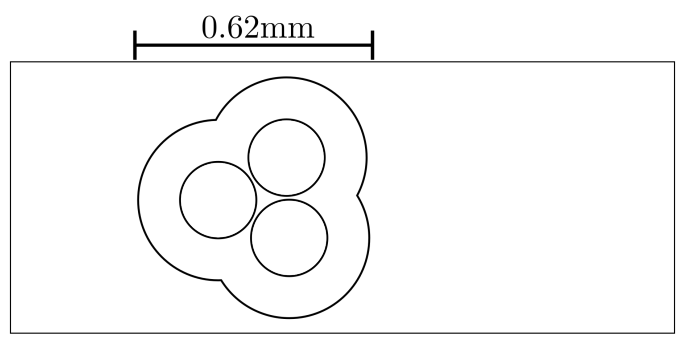

(c) Triangle.

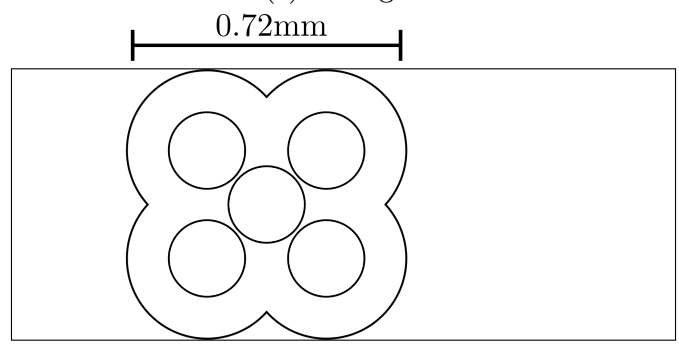

(e) Cross.

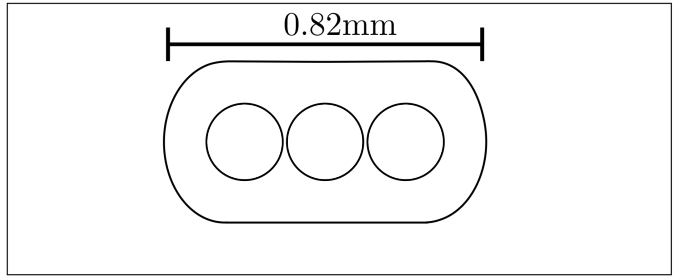

(b) Cylinder. The same as Line except the contours on the droplet are smoothed out and expanded to form a cylindrical body, maintaining hemispherical edges.

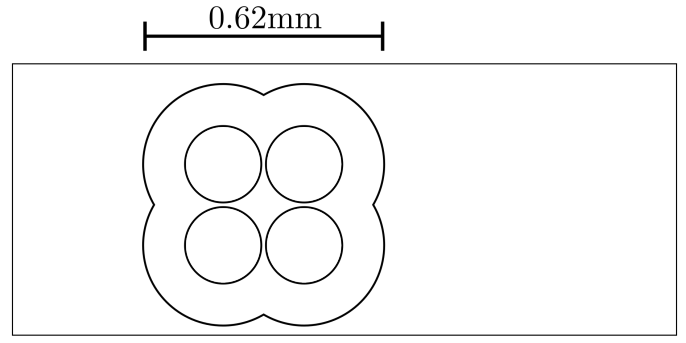

(d) Square

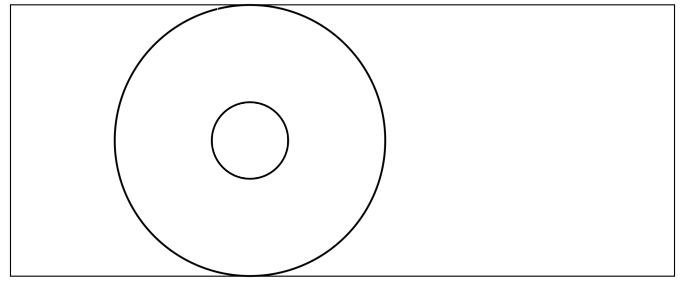

(f) Benchmark.

Figure 5: Top-down visualisations of all of the scenarios explored by the model.

\subsection{Computational modelling}

A Finite Element Method (FEM) is used to simulate the temperature throughout the models. Employing COMSOL Multiphysics 5.5, adaptive meshes are constructed for each given geometry to balance accuracy and computational cost. The computational model discretises the domain into individual elements, the temperature of which can be defined and calculated at each time point of interest. This uses a mixture of triangle and tetrahedral elements, as determined by the solver. A 'fine' element discretisation is employed, which uses a minimum of 2182 tetrahedral elements and 1320 triangular elements. The temperature in the model is calculated over 5 seconds at 0.05 second intervals, a process which runs in a few seconds.

\section{Results}

Samples are considered to be part of the same domain as the droplet, though they are constructed nonetheless. The temperature at these sample points is evaluated, and their average is found. This average temperature is used as a proxy for the overall state of cooling. An alternate measure would be the maximal temperature in the samples. It is possible that within an arrangement of samples, one sample is more at risk of thermal damage than others, and these at risk samples may be more of a concern than other, faster cooling samples. However, in light of current results taking seconds, focus remains on using average temperature. 
Figure 6 shows the average temperature over time of the samples evaluated in four scenarios in which samples are positioned running along the center of the plate. Figure 7 shows the scenarios in which spatial arrangements come from more complex geometries, with a minimum of three samples per scenario. The time taken to reach equilibrium, $77.15 \mathrm{~K}$, in shown in Table 2, for all scenarios, along with calculated cooling rates.

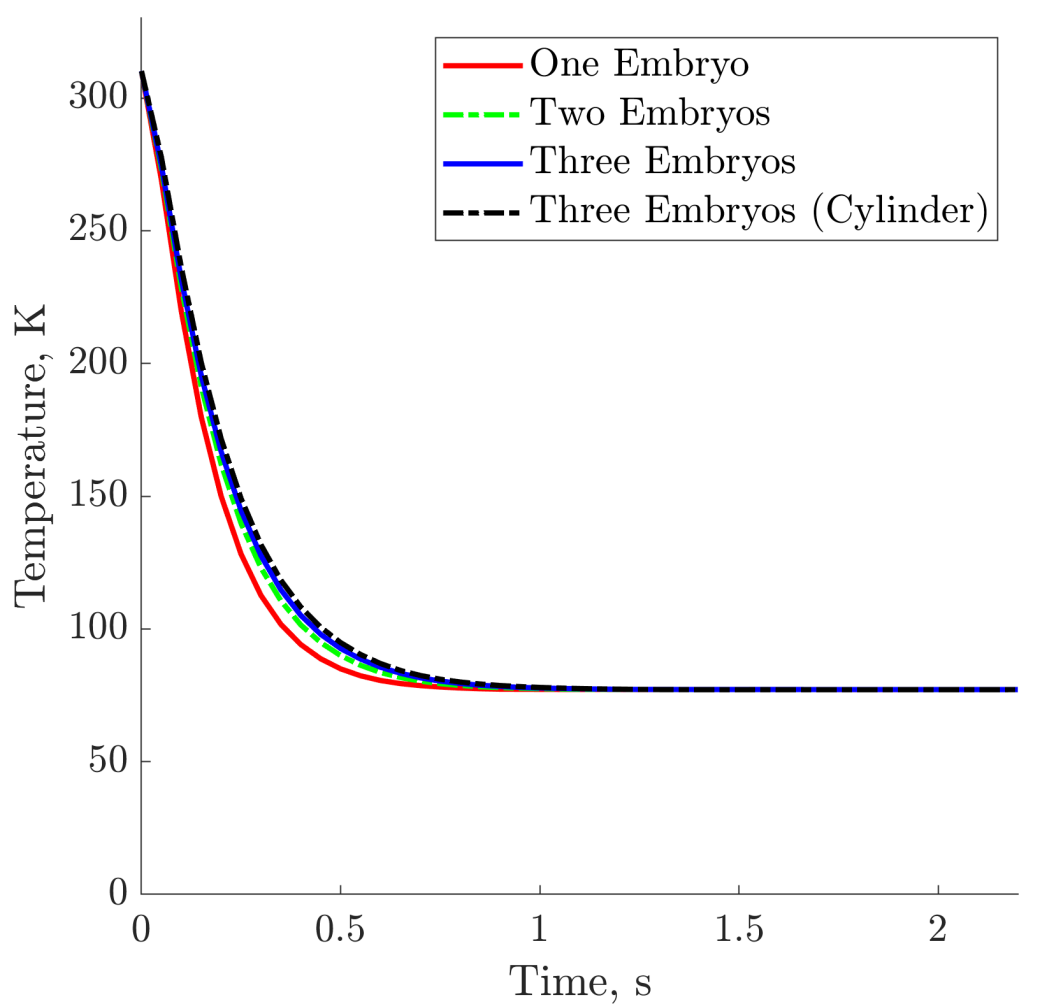

Figure 6: Average temperature over time. The number of embryos increases in a linear format (Figure 5a), with the bounding cylindrical case also presented (Figure $5 \mathrm{~b}$.

\begin{tabular}{|c|c|c|c|}
\hline Model & Number of samples & Vitrification time, $\mathrm{s}$ & Cooling rate, K/min \\
\hline 1 Line & 1 & 1.10 & 12709 \\
\hline 2 Line & 2 & 1.25 & 11184 \\
\hline 3 Line & 3 & 1.35 & 10356 \\
\hline Cylinder & 3 & 1.35 & 10356 \\
\hline Triangle & 3 & 1.35 & 10356 \\
\hline Square & 4 & 1.45 & 9641 \\
\hline Cross & 5 & 1.50 & 9320 \\
\hline Benchmark & 1 & 2.00 & 6990 \\
\hline
\end{tabular}

Table 2: Time taken in seconds for average temperature to reach $77.15 \mathrm{~K}$ inside the embryos.

Figure 6 demonstrates that increasing the number of samples leads to a decrease in the rate at which the temperature changes. The scenarios converge to a single profile, as increasing the length of the line of samples will not affect the rate of cooling of any given sample in the center of the line, as they now lose heat through their contact surfaces with the liquid nitrogen much faster than they would lose heat to the adjacent samples. Hence, evaluating larger collections of samples arranged in a line would not be of value.

Of particular note in Figure 6 is the minor difference between the two cases where there are three embryos. The additional cryoprotectant in the cylinder case slows down cooling of the samples by a small amount, but from Table 2 it can be seen that this difference is on the order of magnitude of 


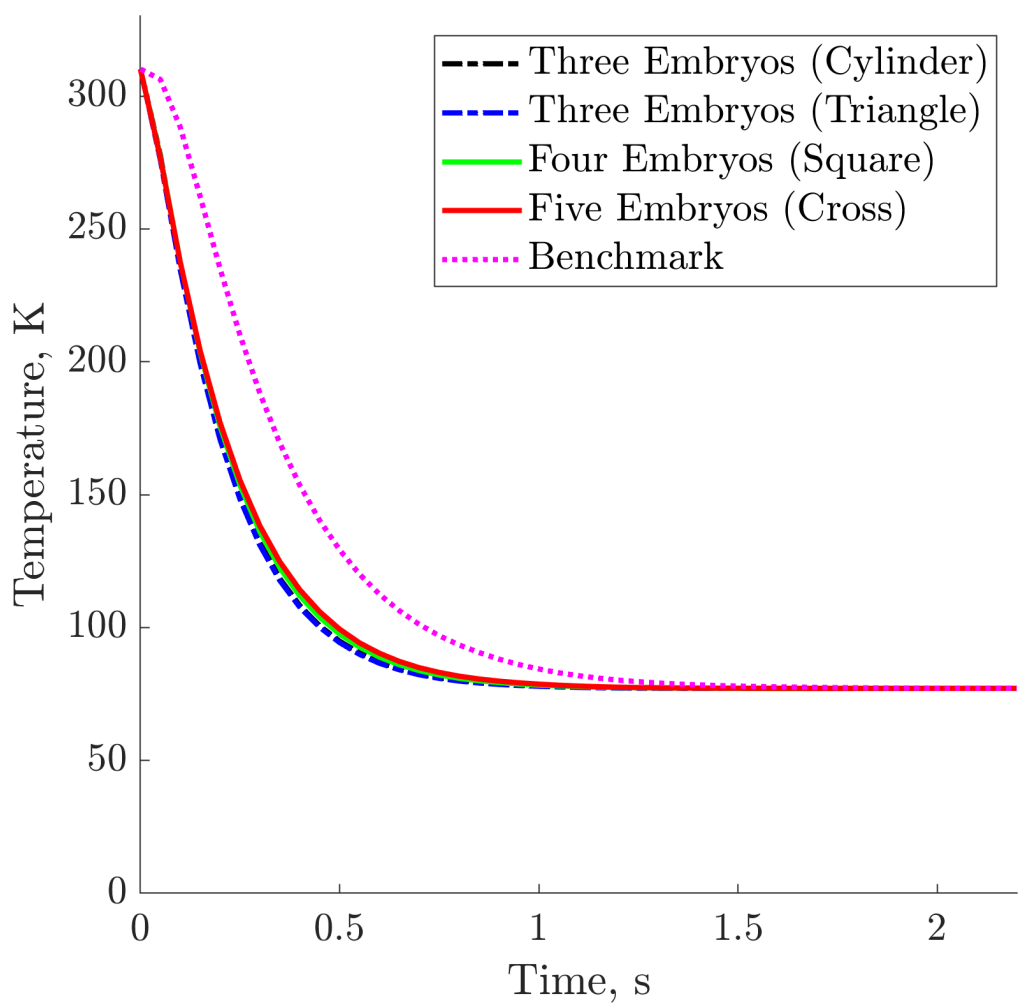

Figure 7: Average temperature over time for different embryo arrangement patterns, depicted in figures $5 \mathrm{c}$. $5 \mathrm{f}$

milliseconds. As such, it can be concluded that these cases act identically, which supports the notion that minor perturbation in droplet shape is unimportant, as hypothesised in assumption 2.2.4.

Figure 7, which shows the temperature profiles in scenarios whose arrangements are not all aligned along the center of the plate, demonstrates a similar effect to Figure 6, where increasing the number of samples leads to a corresponding decrease in the cooling rate. In the two scenarios with three embryos, the cylinder case and the triangle case, the profiles are almost identical, whilst adding more samples to the model increases the time to vitrify.

The temperature profile of the benchmark case in Figure 7 displays the lowest cooling rate of all models. Given that this particular construction is designed to be the most accurate 'worst-case' scenario that is still considered safe to undertake, it can be concluded that any configuration whose cooling rate is greater than that of the benchmark is also a safe and suitable case. As such, all of scenarios tested may be considered safe to use.

It may be noted that the cooling rates in Table 2 are much smaller than those advertised by Kitazato ${ }^{\circledR}$ for this device. Particularly the Kitazato ${ }^{\circledR}$ homepage claims cooling rates of up to $23,000 \mathrm{~K} / \mathrm{min}$, whilst the benchmark case achieves only $6990 \mathrm{~K} / \mathrm{min}$. This implies that the chosen value of $h$ may be too small, which reduces the rate of cooling of the boundaries, and as such the rate at which the samples are cooled. However, this choice of $h$ is justified by literature, and allows us to make a conservative estimate. Despite these assertions, simulations are repeated using a higher value of $h$ in order to make the system more realistic. For these subsequent simulations, $h=10,000 \mathrm{~W} / \mathrm{m}^{2} \mathrm{~K}$ is chosen, which is an order of magnitude greater than the previously chosen parameter, to emphasise the comparison. The results of the simulations are displayed using $h=10,000 \mathrm{~W} / \mathrm{m}^{2} \mathrm{~K}$ in Figures 8 , and tabulate cooling rates in Table 3 .

Comparing Figure 8 to Figures 647, increasing the value of $h$ yields the same pattern of curves, with faster evolution. The cooling rates in Table 3 are still lower than $23,000 \mathrm{~K} / \mathrm{min}$, but are now closer to those expected. All configurations have faster cooling rates than the Benchmark, and differences 


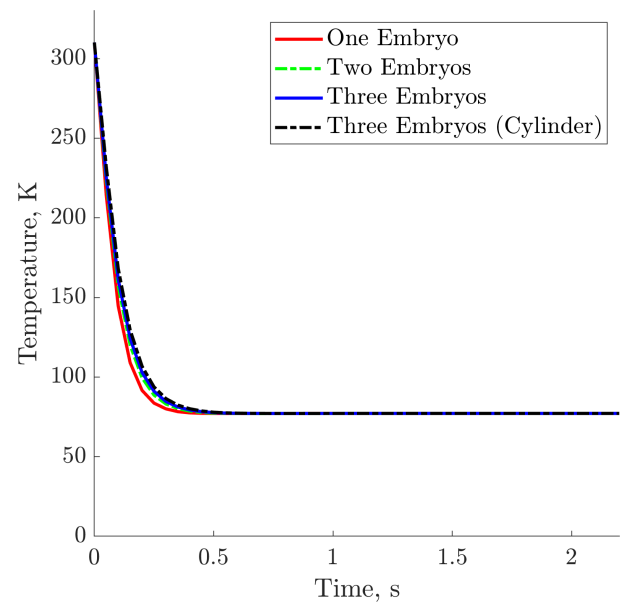

(a) Average temperature over time. The number of embryos increases in a linear format (Figure 5a), with the bounding cylindrical case also presented (Figure $5 \mathrm{~b}$.

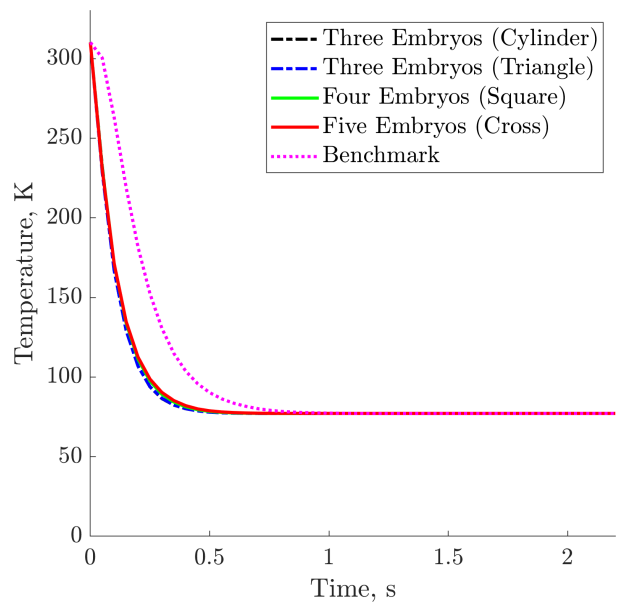

(b) Average temperature over time for different embryo arrangement patterns, depicted in figures $5 \mathrm{c} 5 \mathrm{f}$

Figure 8: Average temperature plots over time, as in Figures 6.7, using the higher surface heat transfer coefficient $h=10,000 \mathrm{~W} / \mathrm{m}^{2} \mathrm{~K}$.

\begin{tabular}{|c|c|c|c|}
\hline Model & Number of samples & Vitrification time, $\mathrm{s}$ & Cooling rate, K/min \\
\hline 1 Line & 1 & 0.55 & 33818 \\
\hline 2 Line & 2 & 0.65 & 21508 \\
\hline 3 Line & 3 & 0.70 & 19971 \\
\hline Cylinder & 3 & 0.75 & 18640 \\
\hline Triangle & 3 & 0.75 & 18640 \\
\hline Square & 4 & 0.8 & 17475 \\
\hline Cross & 5 & 0.85 & 16447 \\
\hline Benchmark & 1 & 1.15 & 12157 \\
\hline
\end{tabular}

Table 3: Time taken in seconds for average temperature to reach $77.15 \mathrm{~K}$ inside the embryos, using the higher surface transfer coefficient $h=10,000 \mathrm{~W} / \mathrm{m}^{2} \mathrm{~K}$.

between models are on the same order of magnitude as those in Table 2 .

\section{Discussion}

The problem of measuring cooling rates during vitrification of multiple oocytes or embryos is an important one, with direct clinical implications, that cannot be addressed through practical experiments. In this model, however, it is shown that differences in cooling rates induced by arrangements of samples are not large enough to pose harm to samples undergoing vitrification. The differences are induced by reducing the surface area exposure of samples to the surrounding liquid nitrogen, leading to a reduction in the rate heat can be transferred to the surrounding liquid nitrogen through the boundaries. So long as droplet volumes remain constant and within manufacturer's limits, all tested arrangements have higher cooling rates than the typical laboratory scenario, which was a 'Benchmark', so therefore are considered valid configurations. This means that there is no need for embryologists to spend precious time moving loaded samples on the plate into particular shapes or arrangements, instead they should focus on aspirating the medium efficiently. This can save precious time for the embryologists working on these samples, and allows them to justify operating to their personal preference, so long as it does not negatively impact survival rates.

These results highlight that when using values of $h$ derived from literature, cooling rates are much smaller than those advertised by Kitazato ${ }^{\circledR}$ for this device. Larger values of $h$ are experimented with, and it can be determined that the results of these simulations are similar to those with lower values 
of $h$, and hence lead to the same conclusions. This can be extended to an infinitely large $h$, which would correspond to a scenario where the boundary instantly attains the temperature of the liquid nitrogen. In this case, the cooling rate is entirely defined by the characteristic timescale reported in Section 2.2.3. As the characteristic length in every scenario except for the benchmark is equal (the height of the droplet), the characteristic timescale is also equal in every scenario, and therefore the time taken to vitrify in every scenario should be approximately equivalent. As such, it is possible to construct both a conservative upper bound, from simulations, and a fast lower bound, using the characteristic timescale, of cooling rates. If the true cooling rates lie somewhere between these two bounds, it can be suggested that as the most extreme cases are safe, so too will be any scenario between these bounds.

These results are dependent on the validity of the assumptions made, however. The assumption in Section 2.2.4 enforces that all droplets must be exact hemispheres, but this may not be the case in reality. Different shaped droplets such as those depicted in[15], or ovoid shapes. In this case, the arrangement of samples may create different shaped droplets between scenarios, which would lead to different characteristic lengths and therefore different cooling rates. Critically, such perturbations to the simulations would lead to small perturbations in the predicted results. These small perturbations would be within the error tolerance of experimental readings and, thus, although these results may not be quantitatively exact, they do offer a robust "rule of thumb" time scale of a couple of seconds for time to vitrification.

The question of how many samples should be mounted to a single device is becoming increasingly important, and this study shows that the only limitation from a thermal perspective is the skill of the embryologist. Specifically, they may mount any number of samples that can be properly stored within $0.1 \mu \mathrm{l}$ of vitrification solution within a reasonable time frame, with suitable in-house validation. This paper does not assess other factors associated with loading of multiple embryos, such as the time taken to process samples, or cost efficiency, instead only validating that loading of multiple samples per device is thermally justifiable. As a result, it cannot conclude that vitrification of multiple samples, or samples from any given arrangement, is completely safe, or optimal. Instead, it can be concluded that thermal differences are an unlikely explanation for variable survival rates arising from variable geometry.

Further work can be done to refine the model further to achieve greater accuracy. This work focuses only on the Open Cryotop ${ }^{\circledR}$ device, but could be replicated for other techniques and devices in common usage. The easiest way to refine these simulations is to revisit the assumptions in Section 2.2. Specifically, the assumptions in Sections 2.2.2 and 2.2.4, around the size and shape of the embryos and droplets, can be easily altered. Small, simple geometries were chosen to explore extreme cases in this study, but these geometries could be further refined to be more accurate and account for realistic droplet shape, more appropriate droplet volumes, and variable embryo location and size within the droplets.

One might wish to address the assumption in section 2.2.1, which suggests liquid nitrogen is a stationary isothermal liquid. In practice, the liquid is boiling, which results in additional mechanisms of temperature change, such as boiling induced convection and other boiling specific effects [33]. The effect of these additional mechanisms between scenarios may differ and may affect cooling rates differently between models. This would involve more complex numerical modelling which would account for fluid motion and phase change. As previously discussed, this assumption is in part accounted for by the choice of $h$, however, so reintroducing fluid motion should not have a large effect on cooling rates.

Further work could also be undertaken to explore the parameter choice in the model. Parameter choices for these simulations are taken from literature [28, 15] and COMSOL, but may not reflect accurate parameter values. Additionally, it was assumed that samples adopt the thermal properties of their surrounding medium. Now that a model has been developed, it is trivial to replace the values 
of the parameters in the model and attempt to derive more accurate outcomes. In particular, the choice of the surface heat transfer coefficient is of interest, to which the system is highly sensitive (see Section 2.2.3). Making a good choice of this parameter is highly important to making the model as accurate as possible. As previously stated, it is likely a greater value of $h$ is needed to match cited cooling rates, which means the models are more strongly dominated by the characteristic timescale, which is equal in all scenarios. As such, the conclusions drawn originally would still hold.

Another important factor to be considered is that some models suggest maximising cooling rates is not the optimum approach to improving survival. Some models of cryopreservation in cells exist 34] that suggest there is some optimal cooling rate range, balanced by the risk of damage to cells from the solute for low cooling rates and the risk of intracellular ice formation for high cooling rates. As such it may not be appropriate to look for arrangements that yield high cooling rates, but instead arrangements that give cooling rates within some optimal interval. Additionally, there is evidence that cooling rates are less significant for the survival of oocytes than warming rates [27]. Indeed, it may even be that some balance between cooling rates and warming rates should be sought. As such, some important future work could be to model the effect of spatial arrangement on the warming process, instead of cooling.

Despite the highlighted shortcomings raised here, results from these simulations are expected to be of the correct order of magnitude. Specifically, so long as the amount of cryoprotectant does not exceed the maximum volume given by the manufacturers guidelines, the cooling process should be on the order of seconds, or less, regardless of the spatial arrangement or number of mounted embryos. As such, so long as one spatial arrangement or setup of samples on a plate is safe with regards to cooling rates, all spatial arrangements are equally safe.

\section{Acknowledgements}

This research is funded by the Knowledge Economy Skills Scholarship (KESS2). KESS2 is a pan-Wales higher level skills initiative led by Bangor University on behalf of the HE sector in Wales. It is part funded by the Welsh Government's European Social Fund (ESF) convergence programme for West Wales and the Valleys.

\section{References}

[1] A. Arav, Y. Natan, Vitrification of oocytes: From basic science to clinical application, in: S. S. Kim (Ed.), Oocyte Biology in Fertility Preservation, Springer, 2015, Ch. 6, pp. 69-84.

[2] W. Rall, G. Fahy, Ice-free cryopreservation of mouse embryos at $-196{ }^{\circ} \mathrm{C}$ by vitrification, Nature 313 (1985) 573-575. doi:10.1038/313573a0.

[3] G. Vajta, L. Rienzi, F. Ubaldi, Open versus closed systems for vitrification of human oocytes and embryos, Reprod. Biomed. Online 30(4) (2015) 325-33. doi:10.1016/j.rbmo.2014.12.012.

[4] M. Kuwayama, G. Vajta, O. Kato, S. Leibo, Highly efficient vitrification method for cryopreservation of human oocytes, Reprod. Biomed. Online 11 (3) (2005) 300-308.

[5] M. Sansinena, M. Santos, N. Zaritzky, J. Chirife, Numerical simulation of cooling rates in vitrification systems used for oocyte cryopreservation, Cryobiology 63 (1) (2011) 32-37. doi: 10.1016/j.cryobiol.2011.04.006. URL https://doi.org/10.1016/j.cryobiol.2011.04.006

[6] S. Seki, P. Mazur, The dominance of warming rate over cooling rate in the survival of mouse oocytes subjected to a vitrification procedure, Cryobiology 59(1) (2009) 75-82. doi:10.1016/j. cryobiol.2009.04.012. 
bioRxiv preprint doi: https://doi.org/10.1101/2021.05.12.443816; this version posted May 14, 2021. The copyright holder for this preprint (which was not certified by peer review) is the author/funder. All rights reserved. No reuse allowed without permission.

[7] S. Seki, P. Mazur, Ultra-rapid warming yields high survival of mouse oocytes cooled to- $196^{\circ} \mathrm{C}$ in dilutions of a standard vitrification solution, PloS one 7 (4) (2012) e36058.

[8] J. Jain, R. Paulson, Oocyte cryopreservation, Fert. Ster. 86(4) 1037-1046.

[9] G. Vajta, P. Holm, M. Kuwayama, P. Booth, H. Jacobsen, T. Greve, H. Callesen, Open pulled straw (ops) vitrification: a new way to reduce cryoinjuries of bovine ova and embryos, Mol. Reprod. Dev. 51 (1) (1998) 53-58.

[10] T. Mukaida, S. Nakamura, T. Tomiyama, S. Wada, C. Oka, M. Kasai, K. Takahashi, Vitrification of human blastocysts using cryoloops: clinical outcome of 223 cycles, Hum. Reprod. 18 (2) (2003) 384-391.

[11] S. Park, E. Kim, J. Oh, H. Nam, K. Lee, S. Park, E. Park, S. Yoon, K. Chung, J. Lim, Ultra-rapid freezing of human multipronuclear zygotes using electron microscope grids, Hum. Reprod. 15 (8) (2000) 1787-1790.

[12] HFEA, Embryo freezing, https://www.hfea.gov.uk/treatments/fertility-preservation/ embryo-freezing/, last accessed on 06/04/2021.

URL https://www.hfea.gov.uk/treatments/fertility-preservation/embryo-freezing/

[13] F. Kleinhans, S. Seki, P. Mazur, Simple, inexpensive attainment and measurement of very high cooling and warming rates, Cryobiology 61 (2) (2010) 231-233. doi:https://doi.org/10.1016/ j.cryobiol.2010.06.011.

URL https://wwW.sciencedirect.com/science/article/pii/S0011224010001094

[14] G. Vajta, M. Kuwayama, Improving cryopreservation systems, Theriogenology 65 (1) (2006) 236-244, iETS 2006 Pre- and Post-Conference Symposia. doi:https://doi.org/10.1016/j . theriogenology.2005.09.026.

URL https://www.sciencedirect.com/science/article/pii/S0093691X05004164

[15] M. Santos., M. Sansinena, J. Chirife, N. Zaritzky, Convective heat transfer coefficients of open and closed cryotop $(\mathrm{R})$ systems under different warming conditions, Cryobiology 84 (2018) $20-26$. doi:https://doi.org/10.1016/j.cryobiol.2018.08.007.

URL http://www.sciencedirect.com/science/article/pii/S001122401830172X

[16] M. Santos, M. Sansinena, J. Chirife, N. Zaritzky, Determination of heat transfer coefficients in plastic french straws plunged in liquid nitrogen, Cryobiology 69 (3) (2014) 488-495. doi : https: //doi.org/10.1016/j.cryobiol.2014.10.010.

URL https://www.sciencedirect.com/science/article/pii/S0011224014006348

[17] K. CORPORATION, Vitrification protocol for cryotop method, https://www.kitazatodibimed.com/wp-content/uploads/CRYOTOP_SAFETYKIT_A5_2019_LOW.pdf, last accessed on 06/04/2021 (2019).

URL

https://www.kitazato-dibimed.com/wpcontent/uploads/CRYOTOP_SAFETYKIT_A5_2019_LOW.pdf

[18] B. Inc, Cryolock - closeed system vitrification device, https://www.biocareeurope. com/images/download/biotech/LL-5005-S-Cryolock-Closed-System-IFU-Rev-C.pdf, last accessed on 06/04/2021 (2019).

URL https://www.biocareeurope.com/images/download/biotech/LL-5005-S-CryolockClosed-System-IFU-Rev-C.pdf

[19] A. Aizer, M. Noach-Hirsh, O. Dratviman-Storobinsky, J. Haas, R. Orvieto, Does the number of embryos loaded on a single cryo-carrier affect post-vitrification survival rate?, Zygote 29 (1) (2021) 87-91. doi:10.1017/S0967199420000453. 
bioRxiv preprint doi: https://doi.org/10.1101/2021.05.12.443816; this version posted May 14, 2021. The copyright holder for this preprint (which was not certified by peer review) is the author/funder. All rights reserved. No reuse allowed without permission.

[20] K. CORPORATION, A device for manipulating biological materials in a process of cryopreservation and a use of such a device, https://patents.google.com/patent/ W02011144352A1/en.

[21] H. Carslaw, J. Jaeger, Conduction of Heat in Solids, Oxford science publications, Clarendon Press, 1986.

URL https://books .google.co.uk/books?id=y20sAAAAYAJ

[22] J. Griffin, B. Emery, I. Huang, C. Peterson, D. Carrell, Comparative analysis of follicle morphology and oocyte diameter in four mammalian species (mouse, hamster, pig, and human), J. Exp. Clin. Assist. Reprod. 3 (1) (2006) 1-9.

[23] L. Veeck, An atlas of human gametes and conceptuses: an illustrated reference for assisted reproductive technology, Taylor \& Francis, 1999.

[24] B. Halim, H. Lubis, D. Novia, M. Thaharuddin, Does oval oocyte have an impact on embryo development in in vitro fertilization?, JBRA. Assist. Reprod. (2017). doi:10.5935/15180557.20170005 .

[25] W. Li, X. Zhou, H. Wang, B. Liu, J. Dai, Heat transfer coefficient of cryotop during freezing, CryoLetters 34 (3) (2013) 255-260.

URL http://europepmc .org/abstract/MED/23812315

[26] T. Wang, G. Zhao, H. Tang, Z. Jiang, Determination of convective heat transfer coefficient at the outer surface of a cryovial being plunged into liquid nitrogen, CryoLetters 36 (4) (2015) 285-288.

[27] P. Mazur, S. Seki, Survival of mouse oocytes after being cooled in a vitrification solution to $-196^{\circ} \mathrm{C}$ at 95 to $70,000^{\circ} \mathrm{C} / \mathrm{min}$ and warmed at 610 to $118,000^{\circ} \mathrm{C} / \mathrm{min}$ : A new paradigm for cryopreservation by vitrification, Cryobiology 62 (1) (2011) 1-7.

[28] Engineering toolbox website, https://www.engineeringtoolbox.com/specific-heat-capacity-d_391.html, last accessed on $06 / 04 / 2021$.

URL https://www.engineeringtoolbox.com/specific-heat-capacity-d_391.html

[29] B. Kleiner, An isoperimetric comparison theorem. Invent. Math. 108 (1) (1992) 37-48. URL http://eudml.org/doc/143987

[30] N. Adam, Use of the Term 'Young's Equation' for Contact Angles, Nature 180 (1957) 809-810. doi:https://doi.org/10.1038/180809a0.

[31] J. Boyard, A. Reignier, S. Chtourou, T. Lefebvre, P. Barrière, T.Fréour, Should artificial shrinkage be performed prior to blastocyst vitrification? a systematic review of the literature and metaanalysis, Hum. Fertil. (2020) 1-9.

[32] B. Fuller, S. Paynter, Fundamentals of cryobiology in reproductive medicine, Reprod. Biomed. Online 9 (6) (2004) 680-691.

[33] E. Roth, Nucleation and heat transfer in liquid nitrogen, Ph.D. thesis, Portland State University (1993).

[34] J. Benson, Mathematical Modeling and Optimization of Cryopreservation in Single Cells, Springer US, New York, NY, 2021, pp. 129-172. doi:10.1007/978-1-0716-0783-1_4. URL https: //doi .org/10.1007/978-1-0716-0783-1_4 